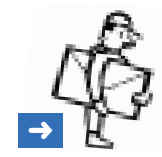

\section{Réflexions ichtyologiques concernant la grippe aviaire}

Ici et là, on lit et on entend des déclarations sur la grippe aviaire de la part de nos autorités, avant tout du conseiller fédéral Couchepin, notre ministre de la santé. Franchement dit, ces déclarations ne m'ont pas donné l'impression que le dossier soit dans les meilleures mains.

Différentes mesures ont certainement été prises, du tamiflu a été stocké par précaution et dans les coulisses, il s'est passé bien plus de choses que nous ne le savons. Par contre, la politique relative à l'acquisition du vaccin suscite mon inquiétude. C'est pourtant clair qu'en cas de pandémie, chaque pays regardera d'abord son propre intérêt. Nous aurions donc grand avantage à produire nous-mêmes ce vaccin. Il faut tout d'abord qu'on m'explique vraiment pourquoi notre firme suisse Berna Biotech est moins apte que d'autres à le faire. Une subvention fédérale de 15 millions permettrait à cette firme de prendre les mesures nécessaires et de procéder immédiatement au développement et à la production de vaccins dès que le virus responsable de la pandémie serait connu. Notre ministre de la santé a déclaré dans la NZZ des 4/5 mars qu' «il était absurde de construire toute une industrie avec des fonds de l'Etat pour un vaccin déterminé, dont on ne sait même pas si on en aura besoin.» (traduction FMH). Mais, nom d'une pipe, qu'avons-nous fait durant de nombreuses années? Monté toute une armée avec des milliards de l'Etat, dont nous ne savons pas non plus si nous en aurons besoin! Construit toute une infrastructure souterraine de bunker dont nous ne savons pas davantage si nous en aurons besoin! En comparaison, le montant de 15 millions est vraiment une somme modique, exactement 2 francs par habitant.

J'ai le sentiment que le problème est sousestimé. Quand Monsieur Zeltner de l'OFSP raconte à la radio qu'en cas de pandémie, le pire scénario possible serait 10000 morts, je m'étonne un peu. En son temps, la grippe espagnole avait causé la mort de 25 à 50 millions de personnes à travers le monde. Si on appliquait ce pourcentage à la population suisse actuelle, le nombre de morts serait au moins dix fois plus élevé. Certes, la situation est aujourd'hui bien différente et il n'est guère possible de faire des pronostics.

Jusqu'à présent, le virus H5N1 a été plein de retenue et a attendu de muter jusqu'à ce que les Suisses aient élaboré leur plan de pandémie. Mais nous ne savons pas s'il continuera à témoigner autant de compréhension pour le cas spécial de la Suisse. Je trouve qu'une certaine hâte serait absolument justifiée dans la préparation d'une production autonome de vaccins. Ne serait-ce pas un sujet pour les médecins politiciens qui siègent au Parlement? Ou pour la FMH?

\section{Dr Thomas Fisch, médecin spécialiste en prévention et santé publique, Riehen}

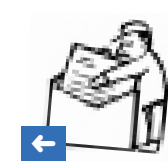

\section{Réplique}

Mon cher Confrère,

Vous avez une manière à peine ingénue de poser la question que tout le monde se pose, voire qui interpelle fondamentalement. Il est bien évident qu'en s'adressant à une entreprise étrangère, en l'occurrence Sanofi-Aventis, franco-allemande, la Suisse est certes bienvenue, mais indépendamment des amabilités d'usage, ne peut en toute honnêteté être considérée comme une cliente d'égale priorité. En cas de pandémie et d'urgences nationales majeures, quand cela pressera vraiment, la France (40 millions d'habitants) et l'Allemagne (100 millions d'habitants) passeront sans doute avant les 7 millions d'Helvètes malgré leur réputation de payer rubis sur l'ongle et même s'ils ont passé leurs ordres avant tous les autres. Le gouvernement français ne s'était-il pas montré d'une conviction supérieure à celle de Novartis lorsqu'il s'agissait de reprendre un fleuron de l'industrie pharmaceutique de l'hexa- 
gone? Fort de cette inquiétude dans l'ordre de la logique politique et de ses aléas humains, un groupe de parlementaires a rencontré les dirigeants de Berna Biotech, dont le capital majoritaire est hollandais, au cours de la session de printemps à Berne. Ces messieurs nous ont déclaré sans la moindre hésitation ni retenue perceptible qu'ils avaient certes une capacité de production suffisante pour faire face aux besoins de la Suisse, qu'à défaut ils auraient pris les dispositions de collaboration nécessaires avec leurs partenaires habituels, mais que ni l'Office fédéral de la santé publique, ni le Conseil fédéral ne leur avaient jamais rien demandé ... A la mesure de l'embarras d'aucun, le sujet est donc loin d'être définitivement clos - les interventions parlementaires sont en cours! Ainsi le poisson n'est pas encore définitivement noyé. Et si la Suisse était membre de l'UE, le problème ne se poseraitil pas différemment?

Dr Yves Guisan, vice-président FMH et conseiller national 